\title{
INFORMATION SUPPORT FOR CRISIS MANAGEMENT IN THE ZLÍN REGION WITH A FOCUS ON METEOROLOGICAL INFORMATION
}

\author{
Miroslav Tomsu \& Robert Pekaj
}
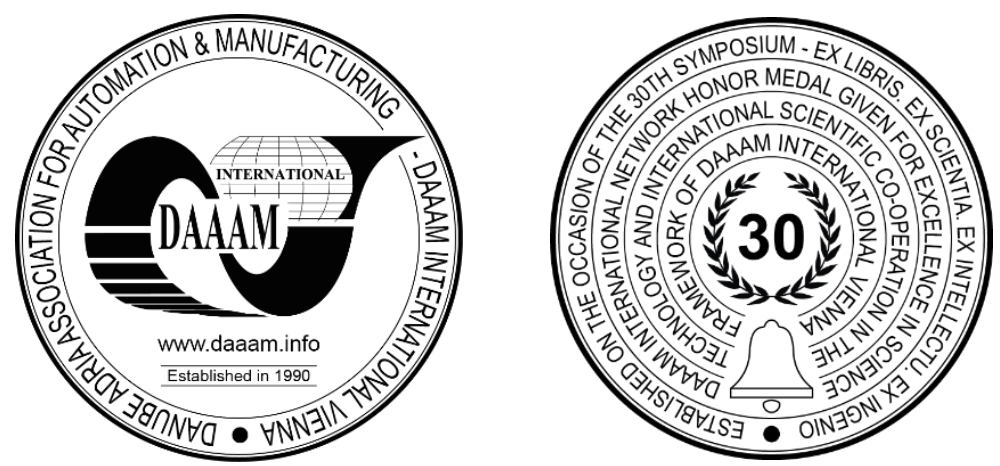

This Publication has to be referred as: Tomsu, M[iroslav] \& Pekaj, R[obert] (2021). Information Support for Crisis Management in the Zlin Region with a Focus on Meteorological Information, Proceedings of the 32nd DAAAM International Symposium, pp.0701-0711, B. Katalinic (Ed.), Published by DAAAM International, ISBN 978-3-90273433-4, ISSN 1726-9679, Vienna, Austria

DOI: $10.2507 / 32$ nd.daaam.proceedings.098

\begin{abstract}
The article deals with information in crisis management, its importance, significance, and effectiveness. Information plays a crucial role in crisis management, as it is a warning and protection of the population. It defines the main requirements for the crisis management information support system and its elements and maps most of the previously used systems, including current and current ones. One of the critical situations in the use of information is the distribution of meteorological information. The article's content is also a presentation of a unique information and communication system in the Zlín region. Finally, the practical use of information in population protection and crisis management will be presented.
\end{abstract}

Keywords: information; information support; crisis management; meteorological information.

\section{Introduction}

All events and phenomena that arise around us, develop and disappear over time, are inextricably linked to a concrete, real and unique space. The space in which this process takes place is called the information space. All the consequences that have occurred in the crisis area have a retroactive effect on that information space and usually change it. information conditions significantly influence every crisis, both in the phase of development of the crisis itself and its solution.

The advent of new technologies also brought new opportunities in the field of information about the territory. The entry of information technology into the field of geodesy, cartography, and geography caused a sharp increase in the possibilities of collecting and processing information about the territory and at the same time opened up a vast space and unprecedented opportunities in their interpretation, modeling, and general use. The advent of digital technologies currently supports the development of spatial simulations that can be used very effectively to model potential phenomena that could occur in crisis situations [1], [2]. 
The aim is to streamline work in the event of emergencies and crisis meteorological situations. The meteor radar will have a significant impact on the early warning of the population in the event of a threat, a faster response in the event of a crisis, which will ultimately reduce the extent of damage to life, health, and property in the event of a crisis.

\section{The importance of information in crisis management}

One of the decisive factors that directly affect the level of crisis management is information. In the conditions of various destructive activities, fast, concise, exhaustive, purposeful, and at the same time, accurate information is invaluable. The resolution of specific crises requires timely notification and warning of the population and continuous information of crisis management bodies about the development of the crisis and the progress of its resolution. Last but not least, it is not possible to solve crises without several various pieces of information necessary to secure people evacuated from crisis areas and units performing rescue and liquidation work.

Information (from the Latin in-formation, formation, rendering) is most often defined as a notification that reduces the degree of uncertainty of the system at the recipient (for example, the recipient/user of the information). Such notification is of practical importance to the recipient, especially its usefulness and importance for solving a particular problem.

There are basically two groups of information requirements:

- General:

- need - unnecessary information unnecessarily overwhelms and makes decision-making more complex,

- truthfulness - the use of false information could cause irreparable damage and reduce confidence in the information provided and its source,

○ completeness - incomplete information is wrong information. Based on it, we can make a completely wrong judgment if we do not have enough relevant information,

- timeliness - outdated information becomes incorrect, untrue information. Ensuring that information is up-to-date is, therefore, a condition for ensuring its integrity.

- Specific - they consist mainly of content, confidentiality, processing, and transmission methods and are usually determined by the contracting authority requesting this information.

Information is a message that one of the possible phenomena from the existing phenomena has occurred, reducing the recipient's ignorance of this phenomenon. But there are several levels of awareness. The following terms characterize information levels:

- Data - are specific images of the properties of the monitored objects, regardless of their more profound meaning and other contexts. These are simple facts expressed in symbols.

- Information - arises from data containing a specific context and purpose. They represent new knowledge for recipients with a specific meaning.

- Knowledge is created by combining information and creative thinking of a person - it is a state of intimate knowledge gained through learning, experience, or association.

The importance of information in crisis management stems primarily from the fact that it is indispensable for all managerial functions and because working with information represents a substantial part of managerial activities in terms of content and time. Information can be perceived as an input assumption and part of crisis management activities [3].

Effective and efficient work with information in crisis management must respect the following principles:

- information processing must be based on an analysis of information needs,

- effective crisis management must have at its disposal essentially the information necessary for the quality solution of the tasks performed,

- the information must be provided in a differentiated and aggregated manner, according to the levels of management,

- information base at the top level should be more oriented towards more comprehensive information on the course and development of the crisis, which is monitored on an ongoing basis, it is necessary to increase the share of the information supplied in real-time,

- the information must be used mainly to support the decision-making process.

The success of executive elements of crisis management depends on the information available to each level of management. Therefore, each manager must formulate, with regard to the conditions of his activity, the information needed for management and constantly update it. This presupposes the creation of a flexible system that would provide the necessary information on the course and development of the situation in the entire external and internal environment of crisis management so that they can be used in preventive activities and in resolving a specific crisis [5]. 


\section{Crisis management information support}

Crisis management information support is a process (set of information activities) supporting information management, decision-making, and cognitive functions. The information need aims to satisfy, through information activities, the information needs necessary for the performance of activities related to crisis management [4].

\subsection{Crisis management information system}

An information system is generally any system that is capable of processing and providing information. In the narrower sense, it is a set of procedures, activities, people, and technologies to collect relevant data, store them until needed, process them to provide answers to a specific set of questions, and finally communicate information to their users. They will take place. The Crisis Act addresses the crisis management information systems in particular, which states that crisis management bodies use crisis management information systems when planning crisis measures and resolving crises. It is further stipulated that the implemented and used crisis management information systems must meet the standards of public administration information systems and the rules:

- transmission of information to superior, subordinate, and cooperating crisis management bodies,

- technical and program adaptation for operation under challenging conditions,

- security of the information stored laid down for the information with the highest degree of classification contained in the processed documentation.

Therefore, the information system is an integral part of crisis management and must be built at the same time as its development, management system, and organizational structure.

The basic mission of a quality information system is to enable easier orientation in complex crisis management processes and to enable the acceleration and improvement of decisions. The role of the information system is to facilitate and improve the decision-making process and to help turn information into decisions on the process of risk analysis, organization of preventive measures, and ensuring the process of resolving specific crises.

The crisis management information system comprises information components (partial information activities) and information elements (individual information means), including their relations, internal and external links, which form an organized unit supporting ensuring protection, defense, internal security, and order.

It consists of the following elements:

- information files,

- information media,

- staff involved in information activities,

- information,

- links between those elements.

The crisis management information system aims to achieve a safe connection of risky objects and localities with monitoring centers, control points, and possible centers of assistance and rescue and mediation of objective reports in resolving crises. The main task of the crisis management information system is the processing, transmission, and use of the necessary information for the effective resolution of crises, including the interconnection of management and executive elements [5], [6].

\section{Software tools to support crisis management}

Each crisis is specific and requires different resolution procedures. For this reason, there is a relatively wide range of software products in our market that deal with this issue. These software tools can be divided as follows:

- accounting, planning, management and combined,

- monitoring,

- computational and modeling,

- analytical,

- special.

Modeling and simulation is an integral part of crisis management, especially in the areas of prevention or prediction of the devastating effects of natural disasters and technological accidents and forecast of the spread of the cause of an emergency (e.g., chemical, biological, and radiation substances, flood wave progression, explosion). Individual model situations are based on a mathematical calculation of the propagation of effects depending on external conditions, such as air temperature, wind direction and strength, surface structure, the size of the source of destructive effects. There are also software products that provide information flows to support communication in resolving a crisis [7]. 


\section{Specific crisis management activities related to SW tools}

\subsection{Analyzes in crisis management of the organization}

Risk analysis is a critical stage in solving the safety and emergency plans of the organization. It should also be part of the project and planning of changes (mergers, change of the company's registered office, development of a new direction of the business, development of a new product, implementation of a new information system, etc.). The entry into this process is the organization's security policy, i.e., the basic strategy of the organization in the field of security, legislative standards, and, among other things, the experience of processors in the field of crisis management. The output is a threat catalog, which serves as input data to the action planning process. A threat is understood as a phenomenon which, provided that at least one threat is realized, can lead to an emergency.

\subsection{Modeling}

Modeling is an integral part of crisis management, especially in prevention, prediction of the devastating effects of natural and technological accidents, and prediction of the spread of the cause of other emergencies (chemical, biological and radiation substances, flood wave progression, explosion, etc.). The products are based on a mathematical calculation of the propagation effects depending on external conditions such as air temperature, wind direction, strength, surface structure, size of the source of destructive effects, etc. The more accurate the required calculation, the more complex and precise the input information required. There are several products whose focus is different.

\subsection{Monitoring}

Regular monitoring is also an integral part of crisis management. Up-to-date knowledge of the situation is essential input information when dealing with an emergency and can be used to detect exceeding the health of a safe condition. Another function that monitoring plays in informing the public.

\subsection{Planning}

Plan - is a binding schedule of procedures for a specific activity (set of measures), which sets out the tasks, forces, places, resources, responsibilities, and timing of the planned move to meet the set objectives [8], [9].

\section{ICWS in the Zlín Region}

ICWS is unique in connecting clients using optical fibers to each crisis staff in the Zlín municipality. The advantage is that the system can also be used in case of a power failure and can communicate with other crisis staff or flood ommissions. ICWS is also part of the meteoradar that monitors weather and precipitation in the municipality.

\subsection{ICWS as a unified platform for crisis management}

The Information, Communication and Warning System (ICWS) of the Zlín Region is a unified communication platform integrating all relevant crisis management workplaces (at the level of the Zlín Region, the Fire and Rescue Service of the Zlín Region and relevant municipalities) into a unified platform at the regional level. Thanks to this, there is a significant strengthening of crisis management tools in the Zlín Region.

The implementation of the project created a modern independent system that allows:

- better crisis prevention and preparedness

- faster response in the event of a crisis

- early warning of the population in the event of a threat

- streamlining the work of the components of the integrated rescue system and crisis management

- reduction of the extent of possible damage to the lives and property of the inhabitants and visitors of the city

- minimizing the duration of the crisis and reducing the costs of intervention

- coordinated elimination of the consequences of the action of harmful factors of crises

- addition of new elements to the system [10]

\subsection{Technical specification of the ICWS central workplace}

ICWS software will control external applications running on the same computer or remote computers on an IP network. To this end, the ICWS software must set up a TCP port (the specific number determined by the configuration) on which it will receive and process commands. Acceptance of the connection may be limited on the server-side to the addresses (or their range) specified during installation. 
After accepting the link, command messages can be sent using the ICWS software. The IS will also receive confirmation and diagnostics from the technology server, both in direct response to the command sent and spontaneously. The VoIP techno-legion will be used as a communication standard to ensure direct voice reporting. The voice compression algorithm currently used in VoIP transmits voice only in a part of the waveband and significantly increases efficiency [11].

\subsection{ICWS server}

The basis of the system will be the server of the central control room ICWS, which enables the control of local systems and data collection through individual access points at the local level (individual municipalities). SW ICWS Server will be created with the help of Microsoft .Net Framework 4.5. The basic architecture of the application will be a three-layer composed of: a presentation layer, an application (domain) layer and a data layer. The data layer will cover access to the Microsoft SQL Server database. The Hibernation Framework will be used for object-relational mapping (ORM).

WPF (Windows Presentation Foundation) technology will be used for the presentation layer. The graphical user interface will be server-side with no interactivity. It will display only the server status, the list of currently connected clients, and presently related access points (individual municipalities). The application (domain) layer will contain the essential functions and logic of the application. Its implementation will not directly link to the presentation or data layer, so a possible future change or modification of the technology at the level of the presentation or data layer will simply be possible. Communication between the ICWS server and client workstations is implemented in networks using the TCP / IP protocol family. The transport layer protocol uses a connection-oriented, reliable TCP (Transmission Control Protocol), which ensures reliability in the event of packet loss by retransmissions, including the rearrangement of received packets (confirming the correct order). The TCP protocol uses port numbers to distinguish between communicating applications. These, together with the IP address, form a so-called socket - unambiguous identification of the endpoint of communication within the network.

An integral part of the server is also data synchronization across connected clients. I.e., in the event of a data change that one client triggers, this change is automatically propagated to other connected clients in the network (without the need for manual refresh, etc.). The same applies to asynchronous events triggered within access points (municipalities) [11].

\subsection{ICWS client}

The client workplace creates an interface for users to control the entire system, monitor the status, and manage it. The ICWS client will be operated at all workplaces of individual municipalities and designated stations within the Zlín Region and the Fire and Rescue Service of the Zlín Region. Communication between the ICWS server and client workstations is implemented in networks using the TCP / IP protocol family. The connection layer protocol is a connection-oriented, reliable protocol TCP (Transmission Control Protocol). In case of packet loss, ensure reliability by repeated sending, including the rearrangement of received parquets (ensuring the correct order). SW ICWS Client will be created with the help of Microsoft .Net Framework 4.5. The basic architecture of the application will be a three-layer composed of: a presentation layer, an application (domain) layer, and a data layer.

The data layer will primarily cover the synchronization of data between the client and the server. Furthermore, the management of locally stored map data and layers, including work with other local files (documents, etc.) WPF (Windows Presentation Foundation) technology will be used for the presentation layer. The graphical user interface is further discussed in the text in individual points. The application (domain) layer will contain the essential functions and logic of the application. Its implementation will not directly link to the presentation or data layer, so a possible future change or modification of the technology within the presentation or data layer will simply be possible.

One of the main functions of the client SW is the presentation of information and the status of the system on the GIS map data of the Zlín Region. "Tiles" of map data and individual layers will be obtained through the WMS service and stored in two-layer caches to achieve maximum rendering speed and the best possible user impression. Firstly, the part in the client's operational memory and the rest on the disk (possibly in the local database) of the client's workplace. Thanks to this system, the map data will be functional even in a connection failure between the client workplace and the GIS server of the Zlín Region. A WMS query for an individual tile will be performed only if it is not in any caching layer. I.e. at the initial request to draw the given "tile" from the client workplace at all. After downloading, the "tile" is permanently stored at the client's workplace. It follows that the data flow between the client and the GIS server of the Zlín Region will be minimal in the long run. At the same time, users will be able to delete locally stored layers (cache) via the user interface and thus force the automatic re-download of these layers from the GIS server [11].

\subsection{ICWS services}

From the user's point of view, the software itself will be based on the application of selected layers from the map base with the possibility of any display and further from the button interface for the transition of individual menus and their subgroups. The display and processing of individual operating and alarm messages will be provided. 
Another functional part of the system will be its communication module to control local exchanges of individual ORPs through their servers for the control and monitoring of integrated systems.

Display of the map layer from the GIS of the Zlín Region:

- $\quad$ Background maps

○ Basic map

- Floodplains

- Orthophoto map with roads, watercourses, descriptions

- Emergency planning zones

Map layers from web services on the Internet:

- Czech Hydrometeorological Institute - reporting profiles - import of water levels

- traffic information, deadlines, accidents, etc.

- Czech Geological Survey - slope instabilities (landslides)

- traffic detours

Map layers from I“'CWS:

- ICWS elements

- Point layers with the possibility of editing:

- Extraordinary events

- Activated / non-activated flood commissions

- Medical facility (capacity)

- Educational facility for evacuation (capacity including meals)

- Gas station

- Objects of possible attack

○ Large poultry farms - the threat of bird flu

All functions listed in the basic requirements for ICWS software, including the static location of units and points of interest, will be displayed in individual layers with a choice. A dynamic change of the status of units in a separate layer of branches, electronic sirens, and control stations or level sensors will be ensured if the VIS system in a specific ORP will support this function.

The SW architecture also consists of a large number of modules:

a) Central control room of the ICWS of the Zlín Region.

b) Videoconferencing systems (interconnection of crisis management participants).

c) Information boards (monitors) for visualization.

d) City camera and surveillance systems.

e) Elements of environmental and chemical detection of hazardous substances.

f) Traffic information.

g) Level sensors on watercourses.

h) Meteorological radar of the Zlín region.

i) Warning information systems.

j) Crisis management documents.

k) Alternative energy sources [11]

\section{Meteoinformation in ICWS}

\subsection{Meteorological radars}

At present, meteorological radars belong to the important and standard equipment of all advanced meteorological services. These devices are known under the general name radar, which originated from the English "RAdio Detection And Ranging". Meteorological radar is a device that is used to detect significant precipitation clouds up to distances of about 100-250 kilometers. In practice, we can encounter the terms large and small meteorological radar. These radars differ not only in size, but mainly in wavelength. Each wavelength size has its letter designation.

- European operational radars (large radars) with a C-band (wavelength $5 \mathrm{~cm}$, frequency $6 \mathrm{GHz}$ ) are suitable for the detection of precipitation clouds in temperate latitudes with a more extended usable range (150-300 km).

- Mobile radars (small radars) with X-band (wavelength $3 \mathrm{~cm}$, frequency $10 \mathrm{GHz}$ ) are suitable for the detection of precipitation clouds in temperate and high latitudes with a smaller usable range $(50-150 \mathrm{~km})[10]$, [11]. 
The MMR50 meteorological radar and the CZRAD network radars have different parameters and properties, which are listed in Table 1:

\begin{tabular}{|c|c|c|c|}
\hline Location & $\begin{array}{l}\text { Holešov industrial } \\
\text { zone }\end{array}$ & Skalky in Protivanov & Brdy-Prague \\
\hline Region & Zlín Region & $\begin{array}{l}\text { South-Moravian } \\
\text { region }\end{array}$ & $\begin{array}{l}\text { Central Bohemian } \\
\text { Region }\end{array}$ \\
\hline Latitude & & $49,501 \mathrm{~N}$ & $49,658 \mathrm{~N}$ \\
\hline Longitude & & $16,790 \mathrm{E}$ & $13,818 \mathrm{E}$ \\
\hline Altitude & $200 \mathrm{~m}$ & $730 \mathrm{~m}$ & $860 \mathrm{~m}$ \\
\hline In operation since & 2014 & 2016 & 2016 \\
\hline Measurement interval & 2 minutes & 5 minutes & 5 minutes \\
\hline Producer & Microstep-MIS & Vaisala & Vaisala \\
\hline Radar type & MMR-50 & Vaisala WRM200 & Vaisala WRM200 \\
\hline Zone & X-band & C-band & C-band \\
\hline \multicolumn{4}{|l|}{ ANTENNA } \\
\hline $\begin{array}{l}\text { Diameter of parabolic } \\
\text { antenna }\end{array}$ & $0.55 \mathrm{~m}$ & $4.5 \mathrm{~m}$ & $4.5 \mathrm{~m}$ \\
\hline $\begin{array}{l}\text { Range of motion in } \\
\text { elevation }\end{array}$ & 1 to $+90^{\circ}$ & 2 to $+92^{\circ}$ & 2 to $+92^{\circ}$ \\
\hline $\begin{array}{l}\text { Number of elevation } \\
\text { angles }\end{array}$ & 13 & 16 & 16 \\
\hline Antenna gain & $32 \mathrm{~dB}$ & $46 \mathrm{~dB}$ & $46 \mathrm{~dB}$ \\
\hline Polarization & Linearly horizontal & $\begin{array}{l}\text { Horizontal / } \\
\text { horizontal + vertical }\end{array}$ & $\begin{array}{l}\text { Horizontal / } \\
\text { horizontal + vertical }\end{array}$ \\
\hline \multicolumn{4}{|l|}{ TRANSMITTER } \\
\hline Frequency & $9.41 \mathrm{GHz}$ & $5.645 \mathrm{GHz}$ & $5.630 \mathrm{GHz}$ \\
\hline Wavelength & $3.0 \mathrm{~cm}$ & $5.3 \mathrm{~cm}$ & $5.3 \mathrm{~cm}$ \\
\hline Transmitter power & $50 \mathrm{~kW}$ & $250 \mathrm{~kW}$ & $305 \mathrm{~kW}$ \\
\hline Repetition frequency & $1500 \mathrm{~Hz}$ & $200-2400 \mathrm{~Hz}$ & $200-2400 \mathrm{~Hz}$ \\
\hline RECEIVER & Linear & Polarization (dual) & Polarization (dual) \\
\hline Receiver sensitivity & $-111 \mathrm{dBm}$ & $-117 \mathrm{dBm}$ & $-117 \mathrm{dBm}$ \\
\hline Dynamic range & $85 \mathrm{~dB}$ & $101 \mathrm{~dB}$ & $101 \mathrm{~dB}$ \\
\hline $\begin{array}{l}\text { Maximum radar } \\
\text { range }\end{array}$ & $100 \mathrm{~km}$ & $260 \mathrm{~km}$ & $256 \mathrm{~km}$ \\
\hline
\end{tabular}

Table 1. Comparison of parameters of mobile radar MMR50 and radars of CZRAD network [13]

In general, it can be said that large radars of the CZRAD network have a higher transmission power, a higher maximum range (up to $250 \mathrm{~km}$ ), and therefore also lower attenuation in precipitation than in the case of small radars. The research of precipitation measurements of the CZRAD radar network and the MMR50 meteorological radar revealed the following findings:

- In both types of meteorological radars (MMR50 and Radar Skalka), the same results were achieved when monitoring precipitation with higher radar reflectivity (from $35 \mathrm{dBZ}$ and higher). This type of precipitation is characteristic of the occurrence of intense showers and storm cores.

- During the passage of precipitation directly above the radar, there was a large attenuation of electromagnetic waves in the precipitation. It has been experimentally proven that the most significant attenuation occurs when detecting snowfall (but also precipitation with very high radar reflectivity above $50 \mathrm{dBZ}$ ) occurring directly above the radar. Due to the significant attenuation, it was then not possible to detect rain at greater distances.

- The low transmission power of $50 \mathrm{~kW}$ for radars operating in the X-band is insufficient to detect collisions with higher radar reflectivity (above $35 \mathrm{dBZ}$ ) arranged in successive bands. These radars can usually detect high collisions with high attenuation with high accuracy so that the rays do not penetrate beyond this collision field. Further precipitation behind this field may no longer be detected, a severe problem in predicting rainfall [13].

\subsection{Meteoradar in the Zlin Region}

In 2015, in addition to the end elements for information, warnings and notifications, a meteorological radar was put into operation within the ICWS project. A meteorological station consisting of its meteor radar located on a mast structure and a control data server is installed in the administrative building of the industrial zone in Holešov. 
The meteor radar is used to obtain information on dangerous meteorological phenomena detectable in the X-zone within a radius of $160 \mathrm{~km}$ and to predict hazardous situations. For the Zlín Region, radio signal coverage means the detection of impending dangerous storm precipitation and torrential rains that directly threaten cities and municipalities in the Zlín Region. The meteoradar transmits the obtained data to the control server to the crisis management workplace (CR). The data are presented using the ICWS software at the CR control workplace in Zlín but also at all ORP workplaces in the Zlín Region.

Meteoradar MMR50 consists of the following main parts:

- support part, with upper rotating part, including cover and random

- antenna

- waveguide part with rotating waveguide coupler

- radar unit

- elevation drive, including sensors

- azimuthal drive, including sensors

- main computer

- complete heating, cooling, and ventilation systems

- power supply and the source part

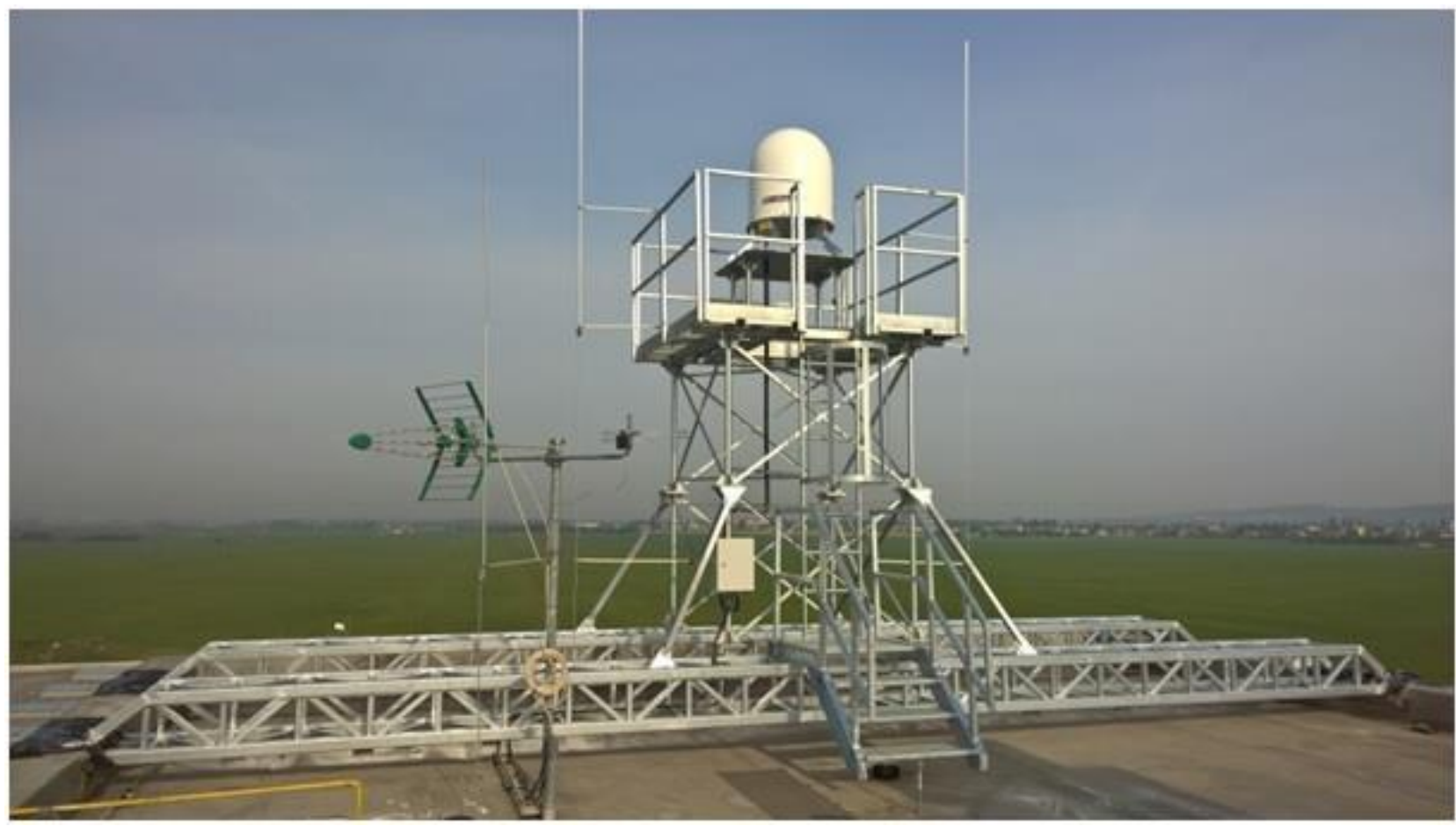

Fig. 1. Meteorological radar of Zlín region [10]

A part of the control computer is the control SW, which enables a fully automatic function of the device without the operator's intervention. It ensures the successful execution of a meteorological measurement, within which it sets up the radar unit and provides the control of the drives. During the measurement, it receives and continuously forwards the measured meteorological data.

The meteorological radar can provide the following functions:

- scanning of backscatter on meteorological objects from the volume of the radar horizon

- editing and assigning data to a spatial matrix

- processing of input data in databases for analyzed and prognostic radar meteorological maps (storms, showers, rain, snowfall, hail, etc.) and distribution of bases towards other systems.

The MMR 50 meteorological radar is a compact device designed to monitor the current state of the earth's atmosphere and obtain information on dangerous meteorological phenomena within a radius of 150 kilometers from the installation site. 


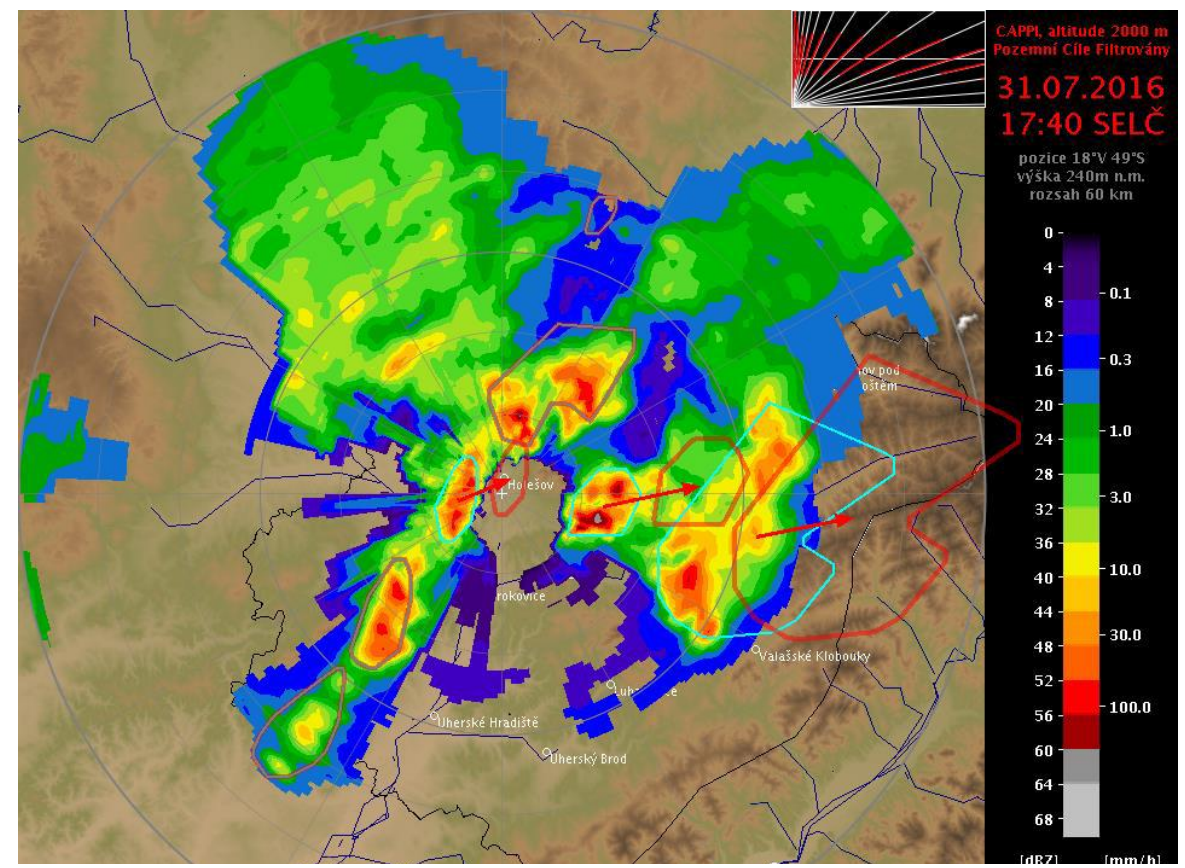

Fig. 2. Measurement of precipitation intensity using the CAPPI $2 \mathrm{~km}$ product from 31.7.2016 [13]

\section{CAPPI module 2 km}

We will use this product to detect and predict intense precipitation and hail, which will most likely fall on the earth's surface and thus can significantly support the occurrence of flash floods.

Color expression of precipitation intensity (radar reflectivity) is the main output information about how intense precipitation occurs at the current time over a given place. To be able to use the information on the current intensity of rainfall to estimate the risk of flash floods, we must monitor the total duration of precipitation over a given locality. In general, the more extended precipitation occurs above a site, the greater the likelihood of flash floods.

\section{VIL module}

The second important product determining the probability of torrential rains and floods is VIL (Vertically Integrated Luquid). The VIL product differs in the $\mathrm{kg} / \mathrm{m} 2$ scale, which is currently intended to estimate the occurrence of torrential rains that can cause flash floods.

In practice, the assessment of the probability of a flash flood will be carried out in the following steps:

- We assign the risk of a flash flood to a given precipitation field according to color.

- Finally, we determine the resulting risk of flash floods at the Radar Information by averaging the probabilities of CAPPI $2 \mathrm{~km}$ and VIL outputs [13].

\subsection{Critical places of implementation}

The software can be implemented in two ways:

- Direct integration into ICWS

- Indirect integration into ICWS

As part of direct integration into ICWS, implementation into the intranet is planned. The application will run on the servers of the Zlín Region. The software is designed to be usable for web and mobile applications and thus meets current rules in software architecture design, including compatibility with existing solutions. One of the critical points could only be an incompatibility with an older version of, for example, the ICWS ZK intranet web interface. The essential point of usability of outputs in practice for the direct variant will then be the login to the intranet, and thus the overall approach to the application. As not all representatives of crisis management and flood planning of municipalities with extended powers in the Zlín Region currently have access, this may mean a specific problem that would need to be solved by another form of access to the ICWS intranet. Indirect integration of the meteor radar into ICWS would only mean that the application would run on servers in the Zlín Region outside the intranet. A particular advantage would be a simplified approach compared to the direct variant both for flood and crisis authorities of municipalities in the Zlín Region and for the public. However, this option would need to be further analyzed in terms of applicability for practical work. 


\subsection{Practical activities when using meteorological radar MMR 50}

The outputs from the MMR 50 meteor radar are used to send warning SMS messages, which inform us about precipitation with an intensity of $20 \mathrm{~mm}$ in 30 minutes and higher within a radius of $5 \mathrm{~km}$. Alert SMS messages are sent only to selected people according to the settings. This setting is still in trial operation, and the "sensitivity" of the setting can be changed.

Here is the general procedure of the crisis management staff using the MMR 50 meteorological radar:

In everyday life:

- Ensure the sending of alerts to the Czech Hydrometeorological Institute.

- Ensure the sending of warning SMS from the MMR 50 radar.

- Ensure a permanent connection to the ICWS and regularly check the operation of the meteor radar.

- Set MMR 50 to "CAPPI" or "VIL" depending on the situation.

\section{When warned by the Czech Hydrometeorological Institute:}

- Inform the crisis management authorities according to the system set up in the relevant municipality.

- Check the outputs from the ALADIN model.

- Check meteor radar outputs

- precipitation intensity estimate (CAPPI),

○ estimation of the amount of liquid water inside the clouds (VIL),

$\circ$ precipitation speed forecast.

When receiving a warning SMS from the MMR 50 meteoradar:

- Study meteoradar data immediately.

- Evaluate meteor radar outputs.

- Evaluate soil saturation from the CHMI Flash Flood Indicator for areas where intense precipitation occurs according to the radar or experience.

- According to the development of the situation, inform the crisis management authorities, check the situation on the spot, organize activities according to the elaborated plans and procedures usual for the relevant ORP with emphasis on the functionality of notifications and warnings [13].

\section{Conclusion}

Information systems systematize the storage of information, their quick retrieval, and many of them have tools that analyze available information and thus provide new information. However, no information system offers a comprehensive proposal for resolving a crisis. The design of the solution is always the subject of a correct and high-quality interpretation of the obtained data and results, which must be performed by an expert, not an information system. ICWS is a unique information and notification system in its scope and concept. Its development is still expected in the future, and I assume that there will be a connection to a similar nationwide system one day.

Through this communication infrastructure, it is possible to use selected services provided by the region. These are expected to expand in the future. The infrastructure also enables connection, via optical fibers, of contributory organizations of the Zlín Region and, last but not least, in cooperation with the Zlín Region, brings opportunities to obtain funds for the construction or development of metropolitan networks in individual municipalities in the region. In this way, information can be disseminated that is valuable primarily to warn the population and protect lives from natural disasters, especially unstable weather.

\section{Acknowledgments}

This work was supported by Grant Project IGA/FAI/2021/003 "SW Population Protection and Soft Target Support" resolved in 2021.

\section{References}

[1] Comfort, L. K.; Sungu, Y.; Johnson, D.; Dunn, M. (2001). Complex systems in crisis: Anticipation and resilience in dynamic environments. Journal of Contingencies and Crisis Management, pp. 144-158.

[2] Hu, Q.; Kapucu, N. (2016). Information communication technology utilization for effective emergency management networks. Public Management Review, pp. 323-348.

[3] Ošmera, P. (1991). Information systems, VUT, ISBN 80-214-0286-5, Brno. 
[4] Lukáš, L.; Hrůza, P.; Kný, M. (2008). Information Management in Security Components. Ministry of Defence of the Czech Republic, ISBN 9788072784608, Prague.

[5] Drozdek, M.; Jelšovská, K. (2013). Information Support of Crisis Management. Silesian University in Opava, Opava

[6] http://www.giswebsite.com/lkc/refs/er/sld001.htm (1995) Lowe WJ. GIS Application Design for an Emergency Management Information System

[7] Adamec, V.; Štolba, L. (2004). Information Technology for Integrated Rescue System and Crisis Management. Public administration online, supplement to the magazine Municipality and finance

[8] Bláhová, M.; Mach, V.; Pavlík, L.; Hromada, M.; Ficek, M. (2019). The Information Security to Software of Crisis Management, Proceedings of the 30th DAAAM International Symposium, pp.1019-1025, B. Katalinic (Ed.), Published by DAAAM International, ISBN 978-3-902734-22-8, ISSN 1726-9679, Vienna, Austria

[9] Víchová, K.; Hromada, M. (2018). Information Support of Crisis Management. Crisis Management - Theory and Practice, pp. 38-45, DOI: 10.5772/intechopen.74094

[10] http://www.colsys.cz, (2017). COLSYS, ICWS of Zlín municipality, Accessed on 2021-09-10

[11] Information, communication and warning system of the Zlín Region (2015). Regional Office of the Zlín Region, Internal document.

[12] Řezáčová, D. et. al. (2007). Physics of clouds and precipitation. 1 ed., Academia ISBN 978-80-200-1505-1, Prague

[13] Šaur D. (2016). Methodology of using the meteorological radar of the Zlín Region for the needs of crisis management. Internal document. 